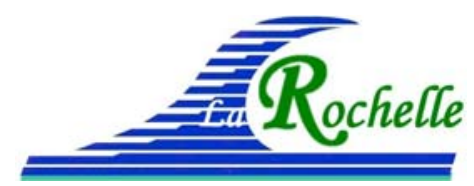

XVèmes Journées Nationales Génie Côtier - Génie Civil La Rochelle, 29 au 31 mai 2018

DOI:10.5150/jngcgc.2018.034 ～(C) Editions Paralia CFL

disponible en ligne - http://www.paralia.fr - available online

\title{
Modélisation des évolutions interannuelles du trait de côte. Processus cross-shore combinés aux processus longshore
}

\author{
Yen Hai TRAN ${ }^{1}$, Eric BARTHÉLEMY ${ }^{1}$ \\ 1. Univ. Grenoble Alpes, CNRS, Grenoble INP, LEGI, 38000 Grenoble, France. \\ Eric.Barthelemy@grenoble-inp.fr
}

\section{Résumé :}

On présente un modèle simple des évolutions interannuelles du trait de côte qui combine à la fois une contribution des processus cross-shore et celle de processus longshore. La modélisation de la contribution longshore repose sur l'approche "oneline" et des propriétés de stationnarité des climats de houle. Les prédictions du modèle sont confrontées à des mesures au pas de temps mensuel des positions du trait de côte de la plage de Narrabeen (Australie). Le forçage par la houle est au pas de temps horaire. Le modèle est en bon accord avec les données qui s'étalent sur 8 ans.

Mots-clés : One-line, Longshore, Cross-shore, Modèle.

\section{Introduction}

La modélisation long-terme (décades) des évolutions transverses (cross-shore) du trait de côte de plages sableuses ne peut pas à l'heure actuelle être effectuée par des modèles décrivant tous les processus aux petites échelles. L'alternative la plus robuste est d'utiliser de la modélisation dite à complexité réduite ou empirique (DAVIDSON \& TURNER, 2009 ; CASTELLE et al., 2014 ; SPLINTER et al., 2014) dans laquelle les processus physiques ne sont pas explicitement résolus et dans laquelle une seule grandeur reflète l'état de la plage. Le plus souvent il s'agit de la position du trait de côte (YATES et al., 2009) mais d'autres descripteurs sont possibles comme le débit sédimentaire cross-shore cumulé (BALDOCK et al., 2017 ; BIRRIEN et al., 2018).

Dans ces modèles l'ensemble des processus cross-shore d'érosion ou d'accrétion sont encapsulés dans un petit nombre de paramètres. La plupart de ces modèles reposent sur le constat phénoménologique qu'une plage tend à relaxer vers un équilibre morphologique (WRIGHT \& SHORT, 1984 ; KRIEBEL \& DEAN, 1993 ; GRASSO et al., 2009). Cet équilibre étant soit représenté par une forme de plage d'équilibre (MILLER \& DEAN, 2004 ; DAVIDSON \& TURNER, 2009 ; BIRRIEN et al., 2018) soit par un climat de houle d'équilibre (DAVIDSON et al., 2013). Les performances de ces modèles dépendent fortement de la qualité des données avec lesquelles les paramètres sont calibrés. Avec de longues séries de données ces modèles cross-shore ont de bonnes capacités prédictives (CASTELLE et al., 2014). Toutefois certains processus ne sont pas pris en compte et en particulier ceux qui induisent de l'érosion ou de l'accrétion à long terme tel que la dérive littorale. 


\section{Thème 2 - Dynamique sédimentaire}

Le transport de sédiments par la dérive littorale ou longshore (KOMAR \& INMAN, 1970) est très important d'un point de vue morphologique. Plus précisément ce sont les gradients de cette dérive littorale qui contrôlent les évolutions de la position du trait de côte (PELNARD-CONSIDÈRE, 1956). Ces gradients sont souvent plus faibles sur des côtes rectilignes que courbes comme sur les plages en baie. Le long de ces dernières les courbures du trait de côte, la diffraction et la réfraction des houles se traduisent par des caractéristiques de la houle au déferlement variables d'un point à un autre de la plage sources de gradients de longshore. Un tronçon de plage en baie sera à l'équilibre si en moyenne dans le temps les vagues sont orthogonales au trait de côte en ce point. Tout forçage dont l'incidence s'écarte de cette direction produira une dérive littorale et comme l'orientation change d'un point à un autre de la plage des gradients de dérive littorale se mettent en place.

Les modèles longshore dits one-line (PELNARD-CONSIDÈRE, 1956) sont les plus classiques. Simples de mise en œuvre quand la dérive littorale est paramétrisée (USACE), ils sont privilégiés en ingénierie (HANSON, 1989) et souvent utilisés pour appréhender qualitativement les modifications induites par des aménagements côtiers. Des approches alternatives ont été proposées pour modéliser la morphogenèse à long terme de la zone littorale. ASHTON et al. (2001) ont étendu l'approche one-line à un modèle cellulaire 2D depuis appelé CEM (Coastal Evolution Model) avec des évolutions récentes par LIMBER et al. (2017) ou HURST et al. (2015) pour mieux rendre compte de la réfraction/diffraction. Des approches hybrides one-line/cross-shore sont aussi proposées (VITOUSEK et al., 2017; ROBINET et al., 2017; ROBINET, 2017).

Il s'agit dans notre étude de prendre en compte les processus long-shore liés aux gradients de dérive littorale dans un modèle d'évolution du trait de côte, nous présentons un modèle simple basé sur une approche "one-line" moyennée dans le temps (REEVE et al., 2004). La partie 2 est consacrée à la présentation du modèle cross-shore utilisé et à l'établissement du modèle original de la contribution longshore aux évolutions du trait de côte. Les parties $3 \& 4$ sont consacrées à la comparaison avec les données de la plage de Narrabeen (Australie).

\section{Le modèle d'évolution du trait de côte}

\subsection{La contribution cross-shore}

La variable pertinente pour classer les profils de plage est le nombre de Gourlay/Dean (WRIGHT \& SHORT, 1984 ; WRIGHT et al., 1985 ; GRASSO et al., 2009). Il est défini par :

$$
\Omega=H_{s} /\left(w_{s} T_{p}\right)
$$




\section{XVèmes Journées Nationales Génie Côtier - Génie Civil \\ La Rochelle, 29 au 31 mai 2018}

où $H_{s}$ est la hauteur significative des vagues, $T_{p}$ la période pic spectral et $w_{s}$ la vitesse de chute des sédiments.

Pour les modèles de type relaxation une plage sableuse tend vers un équilibre si le forçage est invariant dans le temps. Dit autrement pour une plage de profil donné il existe un forçage qui n'induira aucune modification. Ce forçage est représenté par le nombre de Gourlay d'équilibre $\Omega_{e q}$. On postule donc que la vitesse d'évolution du trait de côte s'écrit,

$\frac{d S_{1}}{d t}=F(\Delta \Omega) \quad$ avec $\Delta \Omega=\Omega_{e q}-\Omega$

où $S_{1}(t)$ est la position cross-shore du trait de côte et $\Delta \Omega$ le déséquilibre de forçage qui conditionne l'intensité de la réponse de la plage. On impose à la fonction $F$ de vérifier des propriétés en accord avec ce que l'on comprend du comportement des plages :

- condition 1 si $\Omega=\Omega_{e q}$, le profil de plage ne se modifie pas et donc: $F(0)=0$;

- condition 2 si $\Omega=0$ c'est à dire $H_{s}=0$ (aucune énergie incidente) alors pas de modifications: $F\left(\Omega_{e q}\right)=0$;

- condition 3 si $\Delta \Omega>0$ cela indique que les vagues sont moins fortes que ce qu'il faudrait pour avoir un équilibre: la plage est en accrétion et $F(\Delta \Omega)>0$;

- condition 4 si $\Delta \Omega<0$ cela indique que les vagues sont plus fortes que ce qu'il faudrait pour avoir un équilibre: la plage est en érosion et $F(\Delta \Omega)<0$;

- condition 5 si sur le très long terme la plage n'est ni en érosion ni en accrétion alors l'ensemble des forçages érosifs compensent les forçages accrétifs: $\overline{d S_{1}} / d t=0$, où le signe ( ${ }^{-}$) est la moyenne temporelle d'une variable.

L'étape suivante consiste à faire un développement de Taylor de la fonction $F$ au voisinage de $\Delta \Omega=0$, i.e. au voisinage de $\Omega=\Omega_{e q}$, qui, en tenant compte de la condition 1 et d'un développement à l'ordre 2 , s'écrit:

$F(\Delta \Omega)=b^{ \pm} \frac{\Delta \Omega}{\sigma}+c^{ \pm} \frac{\Delta \Omega^{2}}{\sigma^{2}}$

avec $\sigma$ l'écart type de $\Delta \Omega$. Les coefficients $b^{ \pm}$et $c^{ \pm}$sont à valeurs constantes à déterminer par calibration sur jeux de données. Le développement en série est donc différent suivant que $\Delta \Omega>0$ (signe + pour les coefficients) ou que $\Delta \Omega<0$ (signe -). En effet il est physiquement fondé d'admettre que la fonction $F$ est continue en $\Delta \Omega=0$ mais que la vitesse avec laquelle la plage tend vers l'équilibre par processus érosifs peut ne pas être la vitesse avec laquelle elle tend vers l'équilibre en accrétion. Les conditions 2 , 3 et 4 donnent des relations entre coefficients, pour aboutir au modèle phénoménologique suivant,

$F(\Delta \Omega)=C^{ \pm} \Omega \frac{\Delta \Omega}{\sigma^{2}} \quad$ avec $C^{ \pm}>0$

où $C^{ \pm}$est un paramètre à calibrer qui dépend tout comme $\Omega_{e q}$ du transect de plage considérée et du climat de houle sur celle-ci et qui peut avoir une valeur différente dans 


\section{Thème 2 - Dynamique sédimentaire}

le cas d'une érosion $\left(C^{-}\right)$ou d'une accrétion $\left(C^{+}\right)$. Ce modèle n'est autre que celui de DAVIDSON et al. (2010) similaire à celui de SPLINTER et al. (2014) qui se distingue cependant par un pré-facteur en $P^{1 / 2} \sim H_{s}$ au lieu de $\Omega \sim H / T$ dans (4). La détermination de $\Omega_{e q}$ repose sur la suggestion de WRIGHT et al. (1985),

$\Omega_{e q}=\left[\sum_{i=1}^{2 \Phi} \Omega_{i} 10^{-i / \Phi}\right] /\left[\sum_{i=1}^{2 \Phi} 10^{-i / \Phi}\right]$

où $\Phi$ est interprété comme la "mémoire" dans le temps considérée comme intrinsèque à la plage et son climat de houle. D'un point de vue formel $\Omega_{e q}$ est donc une moyenne pondérée sur une période passée de $2 \Phi$ des forçages $\Omega_{i}=\Omega\left(t=t_{i}\right)$.

La condition 5 est mise en œuvre en distinguant les forçages érosifs $F_{i}^{-}<0$ des forçages accrétifs $F_{i}^{+} \geq 0$. Si sur le long terme il n'y a aucune tendance à l'érosion ou l'accrétion, c'est dire que si la condition 5 est vérifiée (SPLINTER et al., 2014),

$\frac{C^{-}}{C^{+}}=r=-\frac{\overline{F^{+}}}{\overline{F^{-}}}$

où la variable $r$ dépend de $\Phi$ étant donc aussi intrinsèque à la plage. Le coefficient d'érosion vérifie en général $r<1$ ce qui indique que les processus érosifs sont plus intenses que les processus accrétifs. La contribution cross-shore au modèle se formule comme,

$\frac{\mathrm{d} S_{1}}{\mathrm{~d} t}=C\left(F^{+}+r F^{-}\right)$

où $S_{1}$ est la composante cross-shore à la position du trait de côte.

\subsection{La contribution longshore}

A l'équation modèle (7) DAVIDSON et al. (2010) ou SPLINTER et al. (2014), ajoutent une constante pour tenir compte des tendances long terme ou des évolutions non expliquées comme les apports ou puits externes de sédiments. Parmi les phénomènes non pris en compte dans les approches purement cross-shore figurent les processus longshore. Les processus sédimentaires longshore ou longitudinaux sont liés à la dérive littorale induite par le déferlement des vagues en incidence. Les plages en baie avec courbure du trait de côte peuvent être affectées par des gradients de la dérive littorale. Cependant si le climat de houle est d'incidence constante la plage évoluera sur le long terme vers un trait de côte rectiligne parallèle aux crêtes des vagues incidentes. Autrement dit pour une plage à courbure il y a toujours une contribution événementielle (à l'échelle d'une tempête par exemple) du longshore au recul ou avancée du trait de côte. A l'échelle de plusieurs années la forme en plan du trait de côte sera à équilibre si le climat de houle est stationnaire statistiquement (invariance de tous les moments et du spectre directionnel).

Le bilan sédimentaire d'un tronçon longitudinal de plage s'écrit (PELNARDCONSIDÈRE, 1956), 


\section{XVèmes Journées Nationales Génie Côtier - Génie Civil \\ La Rochelle, 29 au 31 mai 2018}

$\frac{\mathrm{d} S_{2}}{\mathrm{~d} t}=-\frac{1}{h_{c}} \frac{\partial Q_{s}}{\partial x}$

où $S_{2}$ est la composante longshore à la position du trait de côte, $h_{c}$ est la profondeur de coupure de la plage (KAMPHUIS, 2010) et $Q_{s}$ est la dérive littorale estimée par la formule dite du CERC (USACE ; REEVE et al., 2004, IDIER et al., 2011):

$Q_{s}=K_{1} H_{b}^{5 / 2} \sin 2 \theta \quad$ avec $\theta=\alpha_{b}(t)-\beta(x)$

où $K_{1} \simeq 0.1$ à $0.2 \mathrm{~m}^{1 / 2} \mathrm{~s}^{-1}$ est une constante qui peut dépendre de la granulométrie des sédiments, de la pente de la plage, de la période de vagues et de la porosité $p \simeq 0.55 \mathrm{du}$ substrat sédimentaire. La plage fait un angle $\beta(x)$ avec l'axe des $x$ (voir fig. 1) et $\alpha_{b}(t)$ est l'angle que font les plans de vagues avec l'axe des $x$ au point de déferlement. Si la plage possède un équilibre moyen sur une longue période de temps alors la moyenne temporelle de l'équation (8) donne,

$\frac{\partial \overline{Q_{s}}}{\partial x}=0$ soit $\overline{Q_{s}}=0$

pour une plage dans une baie, encadrée par des éléments non érodables. On admet que toutes les variables relatives à ce problème se décomposent en une somme d'une moyenne (symbolisée par $(\overline{)})$ et de fluctuations (symbolisée par (')) (REEVE et al., 2004). Cette équation permet de déterminer la forme d'équilibre et les lentes évolutions d'une plage sableuse. Pour cela la moyenne de la dérive littorale $\overline{Q_{s}}$ donnée par la formule du CERC (9) est,

$\overline{Q_{s}}=K_{1} \sin 2 \bar{\theta} \overline{H^{5 / 2} \cos 2 \theta^{\prime}}+K_{1} \cos 2 \bar{\theta} \overline{H^{5 / 2} \sin 2 \theta^{\prime}}$

$\theta=\bar{\theta}+\theta^{\prime}$

Pour une plage en baie on doit avoir,

$\sin 2 \bar{\theta} \overline{H^{5 / 2} \cos 2 \theta^{\prime}}+\cos 2 \bar{\theta} \overline{H^{5 / 2} \sin 2 \theta^{\prime}}=0$

On constate que si les hauteurs de vagues sont dé-corrélées des directions de propagation les quantités $\overline{H^{5 / 2} \sin 2 \theta^{\prime}}$ et $\overline{H^{5 / 2} \cos 2 \theta^{\prime}}$ sont nulles et donc toute valeur de $\bar{\theta}$ est possible: ce sera la valeur initiale. Cependant ce qui caractérise un climat de houle c'est bien qu'il y a une corrélation, éventuellement faible, entre direction et hauteur des vagues. Dans ce cas la solution de (13) est,

$\tan 2 \bar{\theta}=-\frac{\overline{H^{5 / 2} \sin 2 \theta^{\prime}}}{\overline{H^{5 / 2} \cos 2 \theta^{\prime}}} \equiv-\tan \psi$

Physiquement on peut admettre que les fluctuations $\beta$ ' de l'orientation de la plage sont faibles au regard de celles de $\alpha_{b}$ soit que,

$\theta \approx \overline{\alpha_{b}}(x)-\bar{\beta}(x)+\alpha_{b}^{\prime}(x, t)$

La solution de (14), à $\pi$ près, est donc,

$\bar{\beta}(x)=\overline{\alpha_{b}}(x)+\frac{1}{2} \psi(x)$ 


\section{Thème 2 - Dynamique sédimentaire}

L'orientation du trait de côte correspond à l'orientation moyenne des vagues corrigée d'un terme qui mesure le degré de corrélation entre la hauteur des vagues et leurs directions.

Les évolutions du trait de côte impulsées à l'échelle événementielle par les gradients de la dérive littorale découlent de,

$$
\frac{\mathrm{d} S_{2}}{\mathrm{~d} t}=-\frac{K_{1}}{h_{c}} H_{b}^{3 / 2}\left[2 H_{b} \partial_{x} \theta \cos 2 \theta+\frac{5}{2} \partial_{x} H_{b} \sin 2 \theta\right]
$$

Les analyses des données de la plage Narrabeen par HARLEY et al. (2011) (p. 13) indique que $\partial_{x} \theta$ est constant quelle que soit la direction des vagues et que $\partial_{x} H_{b}$ est très faible donc que les produits $H_{b} \partial_{x} \theta$ et $\partial_{x} H_{b}$ sont quasiment des constants.

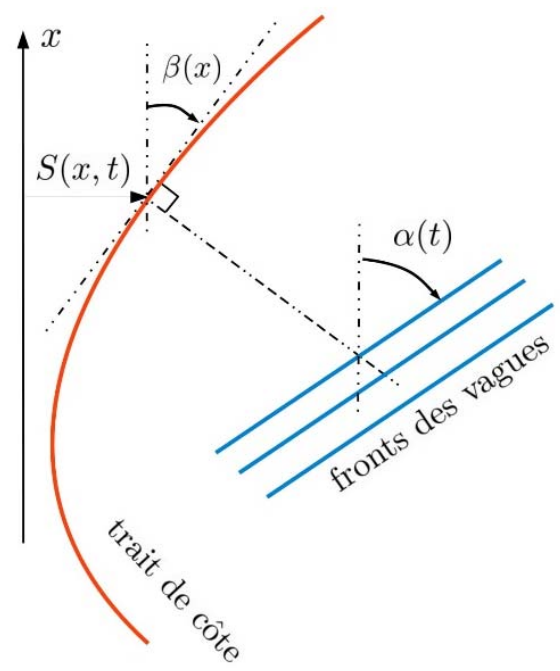

Figure 1. Schéma en plan d'un trait de côte. La convention sur les angles est celle des cartes nautiques. La position du trait de côte est $S$, l'orientation de la plage est donnée par $\beta(x)$ et les plans de vagues/houles ont une orientation donnée par $\alpha$.

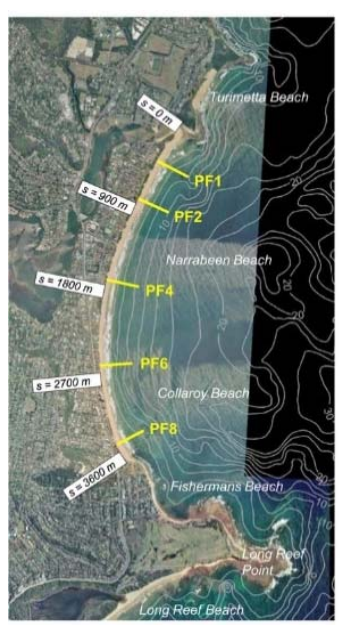

\begin{tabular}{cccccc}
\hline Transect & $\overline{H_{s 10}(\mathrm{~m})}$ & $\sigma_{H_{s 10}}(\mathrm{~m})$ & $\overline{\Omega_{10}}$ & $\sigma_{\Omega_{10}}(\mathrm{~m})$ & $\overline{\alpha_{10}}$ \\
\hline PF1 & 1.15 & 0.5 & 2.56 & 1.05 & $25.61^{\circ}$ \\
PF2 & 0.98 & 0.42 & 2.29 & 0.91 & $21.49^{\circ}$ \\
PF4 & 1.06 & 0.48 & 2.35 & 1.00 & $11.70^{\circ}$ \\
PF6 & 0.94 & 0.45 & 2.10 & 0.99 & $5.04^{\circ}$ \\
PF8 & 0.77 & 0.37 & 1.76 & 0.91 & $-3.01^{\circ}$ \\
\hline
\end{tabular}

Figure 2. A gauche vue aérienne de Narrabeen et repérage des différents transects (d'après TURNER et al. (2016)). A droite: Table 1. Climat des houles à Narrabeen avec 


\section{XVèmes Journées Nationales Génie Côtier - Génie Civil \\ La Rochelle, 29 au 31 mai 2018}

les valeurs moyennes et écart types de: la hauteur significative des vagues $H_{s 10}$ à la profondeur $10 \mathrm{~m}$; le nombre de Gourlay $\Omega_{10}$ à la profondeur $10 \mathrm{~m}$; l'orientation des vagues $\alpha_{10}$ à la profondeur $10 \mathrm{~m}$.

2.3 Le modèle combiné

La forme (17) suggère le modèle de la contribution longshore suivant:

$\frac{\mathrm{d} S_{2}}{\mathrm{~d} t}=H_{b}^{3 / 2}\left[a \cos 2 \alpha_{b}^{\prime}+b \sin 2 \alpha_{b}^{\prime}\right]$

où $a$ et $b$ sont des constantes adimensionnelles. Nous combinons de manière additive (18) et (7) pour obtenir,

$\frac{\mathrm{d} S}{\mathrm{~d} t}=C\left(F^{+}+r F^{-}\right)+H_{b}^{3 / 2}\left[a \cos 2 \alpha_{b}^{\prime}+b \sin 2 \alpha_{b}^{\prime}\right]$

Ce nouveau modèle (TRAN \& BARTHÉLEMY, 2017) combine donc des évolutions du trait de côte $S$ à la fois impulsées par des processus cross-shore et longshore. Les paramètres multiplicatifs $C$, $a$ et $b$ sont déterminés par une méthode d'optimisation ("recuit simulé") qui minimise l'erreur quadratique entre les prédictions des positions du trait de côte par le modèle et celles mesurées. La prédiction des positions $S(t)$ se fait par un schéma explicite au 1er ordre de l'équation différentielle (19).

Table 2. Valeurs des angles donnant les orientations de la plage et des vagues (l'indice 10 indique que tous les estimateurs sont calculés à la profondeur $10 \mathrm{~m}$ ): $\psi 10$ par (14); orientation observée des vagues $\overline{\alpha_{10}}$; prédiction par (16) de l'orientation de la plage : $\overline{\beta_{10}}$; mesure de l'orientation de la plage: $\beta_{m}$.

\begin{tabular}{cccc|c}
\hline Transect & $\boldsymbol{\psi}_{10}$ & $\overline{\alpha_{10}}$ & $\overline{\beta_{10}}$ & $\boldsymbol{\beta}_{\boldsymbol{m}}$ \\
\hline PF1 & $4.80^{\circ}$ & $25.61^{\circ}$ & $28.01^{\circ}$ & $27^{\circ}$ \\
PF2 & $1.08^{\circ}$ & $21.49^{\circ}$ & $22.03^{\circ}$ & $20^{\circ}$ \\
PF4 & $-2.85^{\circ}$ & $11.70^{\circ}$ & $10.28^{\circ}$ & $14^{\circ}$ \\
PF6 & $-5.91^{\circ}$ & $5.04^{\circ}$ & $2.09^{\circ}$ & $-5^{\circ}$ \\
PF8 & $-9.38^{\circ}$ & $-3.01^{\circ}$ & $-7.70^{\circ}$ & $-34^{\circ}$ \\
\hline
\end{tabular}

\section{Application: plage en baie de Narrabeen}

La plage en baie de Narrabeen est située au Nord de Sydney en Australie (TURNER et al., 2016), elle est orientée Nord-Sud face au Pacifique et constituée de sables moyen $(\phi \simeq 2)$. La pente moyenne de la zone de surf est de 1/50 et elle rentre dans la catégorie des plages "intermediate state" ce qui est confirmé par les valeurs de $\Omega$ de la table 1 . Pour cette plage en baie (photo fig.2) on dispose du forçage par la houle entre Janvier 1979 et Octobre 2014 toute les heures à la profondeur de $10 \mathrm{~m}$. Les positions du trait de côte ont été relevées (5 profils en travers) tous les mois entre Avril 1976 et Février 2016. Nous avons choisi comme définition du trait côte la ligne de $z=0.7 \mathrm{~m}$ qui correspond à la hauteur moyenne des marées de vives-eaux. Nous avons extrait de cette base une période de 8 années allant de Janvier 2005 à Décembre 2012 identique à celle 


\section{Thème 2 - Dynamique sédimentaire}

utilisée par SPLINTER et al. (2014). Les caractéristiques moyennes du climat de houle sont synthétisées dans la table 1 .

\section{Résultats et discussion}

Les prédictions des orientations du trait de côte pour chaque transect sont reportées dans la table 2. Les valeurs de références pour la formule (16) sont prises à la profondeur 10 $\mathrm{m}$. On constate un bon accord, sauf pour PF8, entre la formule d'équilibre (16) et l'orientation de la plage $\beta_{m}$ estimée à partir d'images Google Earth. L'erreur sur le transect PF8 est liée à une forte diffraction des vagues par le cap au sud (fig. 2). Une amélioration des prédictions passerait par le calcul de la propagation du champ de houle depuis la profondeur $10 \mathrm{~m}$ jusqu'au déferlement. La correction $\psi$ est faible pour tous les transects sauf pour le PF8. Ceci valide l'hypothèse utilisée dans (17) par laquelle on admet que $\beta(x) \simeq \alpha_{b}$.

Les résultats du modèle combiné (19) sont comparés aux données sur la figure 3. Avant tout notons le signal saisonnier évident avec un cycle d'accrétion en été $\left(\Omega<\Omega_{e q}\right)$ et un cycle d'érosion en hiver $\left(\Omega>\Omega_{e q}\right)$. Notre modèle améliore l'erreur quadratique de $23 \%$ par rapport au modèle de SPLINTER et al. (2014). Ce dernier ne tient pas compte des processus longshore dont l'importance sur la plage de Narrabeen a été analysée par HARLEY et al. (2011). La partie du modèle qui simule les effets longshore prédit pour PF6 une tendance long terme à l'accrétion sur cette période. Notre approche montre que l'on peut avantageusement remplacer la constante additive du modèle de SPLINTER et al. (2014) par une contribution qui prédit les tendances à long terme induites par la dérive littorale. On remarque que la contribution longshore est presque en phase avec l'enveloppe indiquant une réponse sans déphasage de la dérive littorale: au maximum des vagues le longshore prédit un maximum d'érosion. On remarque aussi que la contribution longshore à l'accrétion est en avance de phase par rapport à celle des processus cross-shore. Ce déphasage indique que la dérive littorale peut produire de l'accrétion même au cours d'événements énergétiques.

\section{Remerciements}

Nous sommes reconnaissants du soutien financier de Grenoble INP au travers du projet MEPIERA. Ce travail a été effectué dans le cadre du laboratoire CARE (Ho Chi Minh City) du réseau RESCIF. Le doctorat de Yen Hai TRAN est financé par une bourse d'excellence de l'ambassade de France au Vietnam. Nous tenons aussi à remercier B. CASTELlE, R. ALMAR, P. MARCHESIELlO et T. DUONG pour les échanges scientifiques autour de ce travail. 


\section{XVèmes Journées Nationales Génie Côtier - Génie Civil La Rochelle, 29 au 31 mai 2018}
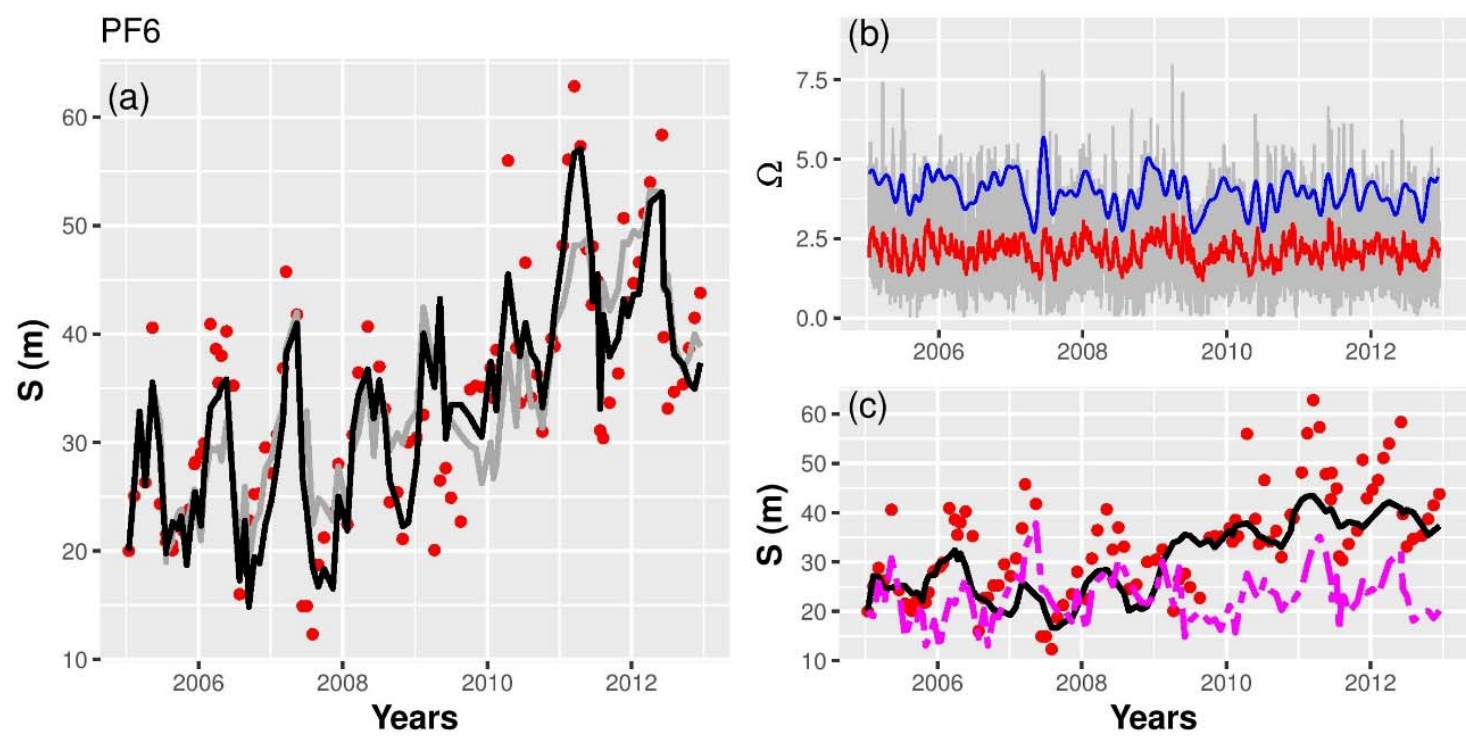

Figure 3. Résultats du modèle combiné pour le transect PF6 de Narrabeen. (a): prédiction du modèle (19) (ligne continue noire) avec une erreur quadratique de 5.13

m; prédiction du modèle de SPLINTER et al. (2014) (ligne grise) avec une erreur quadratique de $6.6 \mathrm{~m}$; cercles rouges: données. (c): contribution longshore du modèle combiné (ligne noire) et contribution cross-shore du modèle combiné (ligne pointtireté). (b): valeurs horaires de $\Omega$ (gris clair); enveloppe supérieure des $\Omega$ (ligne bleue)

et $\Omega_{e q}$ (ligne rouge continue). Les paramètres intrinsèques sont: $\Phi=20$ jours; $r=$ 0.4345. Les paramètres calibrés sont: $C=0.0157 ; a=0.007$ et $b=-0.301$. L'accrétion correspond à une augmentation de $S$.

\section{Références bibliographiques}

ASHTON A., MURRAY A., ARNOULT O. (2001). Formation of coastline features by large-scale instabilities induced by high-angle waves. Nature, Vol. 414(6861), pp 296. https://doi.org/10.1038/35104541

BALDOCK T., BIRRIEN F., ATKINSON A., SHIMAMOTO T., WU S., CALLAGHAN D., NIELSEN P. (2017). Morphological hysteresis in the evolution of beach profiles under sequences of wave climates-part 1; observations. Coastal Engineering, Vol. 128, pp 92-105. https://doi.org/10.1016/j.coastaleng.2017.08.005

BIRRIEN F., ATKINSON A., SHIMAMOTO T., BALDOCK T. (2018). Hysteresis in the evolution of beach profile parameters under sequences of wave climates-part 2; modelling. Coastal Eng., Vol. 133, pp 13-25. https://doi.org/10.1016/j.coastaleng.2017.12.001

CASTELlE B., MARIEU V., BUJAN S., FERREIRA S., PARISOT J., CAPO S., SÉNÉCHAL N., CHOUZENOUX T. (2014). Equilibrium shoreline modelling of a high-energy meso-macrotidal multiple-barred beach. Marine Geology, Vol. 347, pp 85-94. https://doi.org/10.1016/j.margeo.2013.11.003 


\section{Thème 2 - Dynamique sédimentaire}

DAVIDSON M., TURNER I. (2009). A behavioral template beach profile model for predicting seasonal to interannual shoreline evolution. Journal of Geophysical Research: Earth Surface, Vol. 114(F1).

DAVIDSON M., LEWIS R., TURNER I. (2010). Forecasting seasonal to multi-year shoreline change. Coastal Engineering, Vol. 57(6), pp 620-629. https://doi.org/10.1016/j.coastaleng.2010.02.001

DAVIDSON M., SPLINTER K., TURNER I. (2013). A simple equilibrium model for predicting shoreline change. Coastal Engineering, Vol. 73, pp 191-202. https://doi.org/10.1016/j.coastaleng.2012.11.002

GRASSO F., MICHALLET H., BARTHÉLEMY E., CERTAIN R. (2009). Physical modeling of intermediate cross-shore beach morphology: Transients and equilibrium states. Journal of Geophysical Research : Oceans, Vol. 114(C9).

HANSON H. (1989). GENESIS: A generalized shoreline change numerical model. Journal of Coastal Research, pp 1-27.

HARLEY M., TURNER I., SHORT A., RANASINGHE R. (2011). A reevaluation of coastal embayment rotation: The dominance of cross-shore versus alongshore sediment transport processes, collaroy-narrabeen beach, southeast australia. Journal of Geophysical Research: Earth Surface, Vol. 116(F4).

HURST M., BARKWITH A., ELLIS M., THOMAS C., MURRAY A. (2015). Exploring the sensitivities of crenulate bay shorelines to wave climates using a new vector-based one-line model. Journal of Geophysical Research: Earth Surface, Vol. 120(12), pp 2586-2608. https://doi.org/10.1002/2015JF003704

IDIER D., FALQUÉS A., RUESSINK B., GARNIER R. (2011). Shoreline instability under low-angle wave incidence. Journal of Geophysical Research: Earth Surface, Vol. $116(\mathrm{~F} 4)$.

KAMPHUIS J. (2010). Introduction to coastal engineering and management, volume 30. World Scientific. https://doi.org/10.1142/7021

KOMAR P., INMAN D. (1970). Longshore sand transport on beaches. Journal of geophysical research, Vol. 75(30), pp 5914-5927. https://doi.org/10.1029/JC075i030p05914

KRIEBEL D., DEAN R. (1993). Convolution method for time-dependent beach-profile response. Journal of Waterway, Port, Coastal, and Ocean Engineering, Vol. 119(2),; pp 204-226. https://doi.org/10.1061/(ASCE)0733-950X(1993)119:2(204)

LIMBER P., ADAMS P., MURRAY A. (2017). Modeling large-scale shoreline change caused by complex bathymetry in low-angle wave climates. Marine Geology, Vol. 383, pp 55-64. https://doi.org/10.1016/j.margeo.2016.11.006

MILLER J., DEAN R. (2004). A simple new shoreline change model. Coastal Engineering, Vol. 51(7), pp 531-556. https://doi.org/10.1016/j.coastaleng.2004.05.006 PELNARD-CONSIDĖRE R. (1956). Theoretical tests on the shoreline evolution of sand and gravel beaches. In 4th Journées de l'Hydraulique, pp 289-298. 


\section{XVèmes Journées Nationales Génie Côtier - Génie Civil La Rochelle, 29 au 31 mai 2018}

REEVE D., CHADWICK A., FLEMING C. (2004). Coastal engineering: processes, theory and design practice. CRC Press.

ROBINET A., CASTELlE B., IDIER D., MARIEU V., SPLINTER K., HARLEY M. (2017). On a reduced-complexity shoreline model combining cross-shore and alongshore processes. In Coastal Dynamics 2017.

ROBINET A. (2017). Modélisation de l'évolution long-terme du trait de côte le long des littoraux sableux dominés par l'action des vagues. PhD thesis, Bordeaux

SPLINTER K., TURNER I., DAVIDSON M., BARNARD P., CASTELLE B., OLTMAN-SHAY J. (2014). A generalized equilibrium model for predicting daily to interannual shoreline response. Journal of Geophysical Research: Earth Surface, Vol. 119 (9), pp 1936-1958. https://doi.org/10.1002/2014JF003106

TRAN Y., BARTHÉLEMY E. (2017). Long-term shoreline evolution modeling in embayed beaches. In Coastal Dynamics 2017.

TURNER I., HARLEY M., SHORT A., SIMMONS J., BRACS M., PHILLIPS M., SPLINTER K. (2016). A multi-decade dataset of monthly beach profile surveys and inshore wave forcing at narrabeen, australia. Scientific data, 3. https://doi.org/10.1038/sdata.2016.24

CERC USACE. Shore protection manual. US Army Corps of Engineers, Coastal Engineering Research Center, Vicksburg, MS.

VITOUSEK S., BARNARD P., LIMBER P., ERIKSON L., COLE B. (2017). A model integrating longshore and cross-shore processes for predicting long-term shoreline response to climate change. Journal of Geophysical Research: Earth Surface, Vol. 122 (4), pp 782-806. https://doi.org/10.1002/2016JF004065

WRIGHT L., SHORT A. (1984). Morphodynamic variability of surf zones and beaches: a synthesis. Marine geology, Vol. 56(1), pp 93-118. https://doi.org/10.1016/0025-3227(84)90008-2 WRIGHT L., SHORT A., GREEN M. (1985). Short-term changes in the morphodynamic states of beaches and surf zones: an empirical predictive model. Marine geology, Vol. 62(3-4), pp 339-364. https://doi.org/10.1016/0025-3227(85)90123-9

YATES M., GUZA R., O'REILLY W. (2009). Equilibrium shoreline response: Observations and modeling. Journal of Geo-physical Research: Oceans, Vol. 114(C9). https://doi.org/10.1029/2009JC005359 
Thème 2 - Dynamique sédimentaire 